\title{
Star - Gas Cycle in Galaxies
}

\author{
Jan Palouš \\ Astronomical Institute, Academy of Sciences, Boční II 1401, 14041 Prague 4, Czech Republic \\ email: palous@ig.cas.cz
}

\begin{abstract}
The importance of the star-gas cycle for the evolution of galaxies is discussed. We describe three different ways of stellar mass loss: a) the gas return from low and intermediate mass stars, b) the stellar winds from OB associations; c) the superwinds from superstar clusters.
\end{abstract}

Keywords. supernovae: general, stars: winds, ISM: abundances, ISM: structure

\section{Introduction}

The gas in galaxies is consumed by star formation. Looses are partially compensated by gas accretion from galaxy vicinity, see van der Hulst \& Sancisi (2004). A nearby example of the gas accreted to the gaseous disk of the Milky Way is the Magellanic stream of neutral HI floating out of the satellite galaxies, Small and Large Magellanic Clouds, see Brüns et al. (2004). Gas accretion has important consequences to galaxy evolution. It influences the metalicity of the disk offering a possible solution to the G-dwarf problem. Fresh accreted gas may trigger new episodes of spiral waves and help with the reconstruction of central bars. The infalling gas may be used for disk reformation when it was damaged by merger events.

\section{Three ways from stars to the ISM}

Another part of the consumption is compensated by gas recycling: a fraction of gas, which is enriched with the products of stellar evolution, is returned to the interstellar medium as stellar wind and in supernova explosions. The returned mass and energy structure the ISM driving the supersonic turbulence. In this contribution we distinguish three ways of the gas recycling, see Fig. 1: a) slow and long lasting return from low and intermediate mass stars; b) the stellar winds of massive stars in OB associations; and c) the superwinds blowing from superstar clusters.

\subsection{Low and Intermediate Mass Stars}

The influence of the continuous mass loss from low and intermediate mass stars on the evolution of galaxies is described by Jungwiert et al. (2001). The galactic bars dissolve as a consequence of deflections of stellar orbits on the central mass concentration in combination with a gravity torques due to a phase shift between gaseous and stellar flow lines, see Bournaud et al. (2005). The mass and energy return from stars form structures near resonant orbits, reduces the torque between gas and stars extending bar lives, and enhances gas flow to the central region Jungwiert (this volume).

\subsection{OB Associations}

The young stars in OB associations insert mass and energy into the ISM as stellar winds and in supernova explosions. They form expanding structures and drive the supersonic turbulence. The bubbles may break through the dense parts of the galactic disk, see 


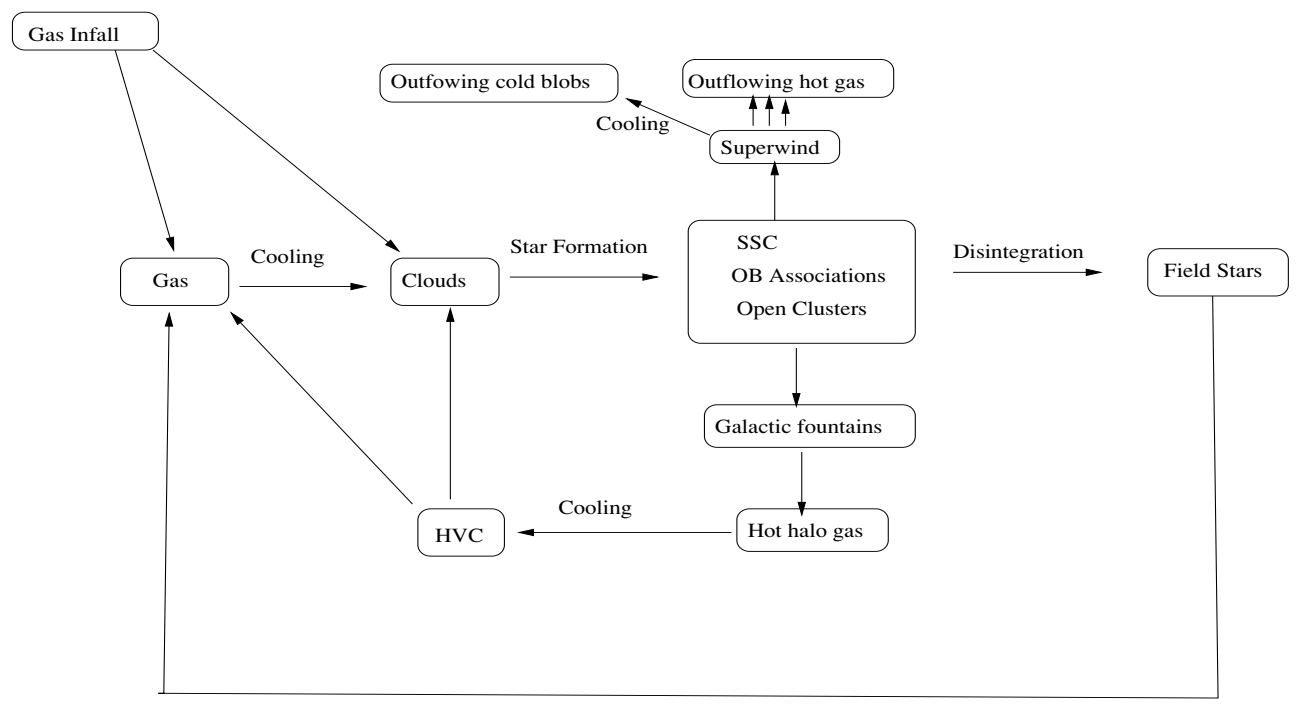

Figure 1. The star - gas cycle in galaxies

Efremov et al. (1999). The hot gas enhances the metalicity of the galactic halo and forms the galactic fountains that return the gas to the galactic plane as high velocity clouds.

\subsection{The Superstar Clusters}

Galaxy collisions such as NGC 6621/2 lead to formation of Superstar Clusters (SSC), see Zhang et al. (2001). Their masses range from $10^{4}$ to a few $10^{6} \mathrm{M}_{\odot}$ with mean effective radii $\sim 4$ pc and ages a few Myr. They reside in starburst galaxies such as M82, see de Grijs et al. (2001). The winds and supernova explosions cause a large overpressure, which results from the thermalization of the deposited energy in random collisions, forming a superwind able to leave the galaxy and provide the products of stellar evolution to intracluster space, see Tenorio-Tagle et al. (2006).

\section{Conclusions}

The SSC, OB associations and the long-lived low and intermediate mass stars recycle the gas enriched by stellar evolution. They provide metals to the intracluster medium, they form galactic fountains, shells and supershells, they influence the galaxy evolution and help to concentrate the mass to the galactic central regions.

Acknowledgments I acknowledge the Institutional Research Plan AV10030501 of the Astronomical Institute, Academy of Sciences of the Czech Republic, and the project LC 06014 Center for Theoretical Astrophysics.

\section{References}

Bournaud, F., Combes, F. \& Semelin, B. 2005 MNRAS 364, 18.

Brüns, C., Kerp, J., Staveley-Smith, L., et al. 2004 A\&SA 432, 45.

de Grijs, R., O'Connell, R. W. \& Gallagher, J. S. 2001 ApSS 276, 397.

Efremov, Yu. N., Ehlerová, S. \& Palouš, J. 1999 A 6 A 350, 457.

Jungwiert, B. 2006 this volume.

Jungwiert, B., Combes, F. \& Palouš, J. 2001 A 6 A 376, 85.

van der Hulst, T. \& Sancisi, R. 2004 IAU Symp. 217, 122.

Tenorio-Tagle, G., Wünsch, R., Silich, S. \& Palouš, J. 2006 ApJ submitted.

Zhang, Q., Fall, S. M. \& Whitmore, B. C 2001 ApJ 561, 727. 


\section{Discussion}

Dimitri Gadotti: I have a comment. We have been trying to estimate bar ages and found that young bars are found preferentially in disk dominated galaxies, gas rich and with star formation. Thus recycling of bars should happen more often in these galaxies, fitting well into your results.

JAN PALOUŠ: Thanks 